\title{
A Path-Based Gradient Projection Algorithm for the Cost-Based System Optimum Problem in Networks with Continuously Distributed Value of Time
}

\author{
Wen-Xiang $\mathrm{Wu}^{1}$ and Hai-Jun Huang ${ }^{2}$ \\ ${ }^{1}$ Beijing Key Lab of Urban Intelligent Traffic Control Technology, North China University of Technology, Beijing 100144, China \\ ${ }^{2}$ School of Economics and Management, Beihang University, Beijing 100191, China \\ Correspondence should be addressed to Wen-Xiang Wu; wwx816@gmail.com
}

Received 30 November 2013; Accepted 11 February 2014; Published 20 March 2014

Academic Editor: Ching-Jong Liao

Copyright ( $\odot 2014$ W.-X. Wu and H.-J. Huang. This is an open access article distributed under the Creative Commons Attribution License, which permits unrestricted use, distribution, and reproduction in any medium, provided the original work is properly cited.

\begin{abstract}
The cost-based system optimum problem in networks with continuously distributed value of time is formulated as a path-based form, which cannot be solved by the Frank-Wolfe algorithm. In light of magnitude improvement in the availability of computer memory in recent years, path-based algorithms have been regarded as a viable approach for traffic assignment problems with reasonably large network sizes. We develop a path-based gradient projection algorithm for solving the cost-based system optimum model, based on Goldstein-Levitin-Polyak method which has been successfully applied to solve standard user equilibrium and system optimum problems. The Sioux Falls network tested is used to verify the effectiveness of the algorithm.
\end{abstract}

\section{Introduction}

The traffic assignment problem consists in determining which routes to assign to the drivers who travel on a transportation network from some origins and some destinations. Wardrop [1] stated two principles for determining the assignment. The first principle leads to an equilibrium state in which no user can reduce his travel time by using an alternative route. The second principle induces a system optimal state in which the total travel time of all users (or the average journey time in a network) is minimum regarding the benefit of the society (i.e., whole traffic). It is well known that, in the standard traffic network equilibrium model, a so-called marginal-cost toll (equivalent to the negative externality that an additional individual imposes on other users of the system) can drive a user equilibrium flow pattern to a system optimum [2]. This conventional traffic network equilibrium model typically assumes that users' VOTs are identical, that is, homogeneous users. However, user heterogeneity is manifested in the fact that some travelers take slower paths to avoid tolls while others choose tolled roads to save time. Some studies pointed out that the VOT varies significantly across individuals because of different socioeconomic characteristics, trip purposes, attitudes, and inherent preferences [3-6].

The concept of value of time (VOT) plays a central role in road pricing analysis as it describes how users make tradeoffs between money and time in response to road toll charges. The network disutility can be measured in travel time or travel cost. It is obvious that different system optimal (SO) flow patterns will be obtained if we use different units (time or money) to measure the system disutility. Previous studies that address user heterogeneity can be classified into two categories [7]. The first is the multiclass approach in which the entire feasible VOT range is divided into several predetermined intervals according to a discrete VOT distribution or some socioeconomic characteristics $[2,8-10]$. The second lets VOTs be continuously distributed across the population of trips, which is regarded to be more rational than others [11-15]. In the these studies, including those dealing with tworoute or highway/transit two-mode problems [12, 16-19], the continuously distributed VOT between each OD pair was treated as a random variable following a probability density function $[13,15,20-22]$. 
For more realistically capturing the travelers' path choice behavior in response to toll charges, Wu and Huang [23] assumed that the VOT varies significantly across individuals because of their different socioeconomic characteristics, trip purposes, attitudes, and inherent preferences. Furthermore, the VOT of each user is assumed to be deterministic and constant, because the factors influencing VOT keep unchanged within a certain time period. They extended the work of Yang and Huang [2] to the case with continuously distributed value of time across users, for finding anonymous tolls to realize target flow pattern in networks with continuously distributed value of time. To find anonymous tolls to realize system optimum in cost units, Wu and Huang [23] proposed a cost-based system optimum model in general networks with continuously distributed value of time but did not study its traffic assignment algorithms. The calculation of the model is very difficult [24]. It is formulated in a path-based form and cannot be solved by the Frank-Wolfe algorithm which is linkbased and cannot provide path flows.

In the past, path-based algorithms, even those not enumerating all possible paths, were traditionally discarded by transportation researchers for solving large-scale network problems because of intensive memory requirements and the difficulties in manipulating and storing paths [25]. However, path-based algorithms automatically provide not only the link-flow solution but also the path-flow solution that may be required in certain applications. In light of magnitude improvement in the availability of computer memory in recent years, recent research on path-based algorithms has demonstrated and established that it is a viable approach for traffic assignment problems with reasonably large network sizes [26-29]. Chen et al. [25] stated that much of the attention has been focused on two particular algorithms: the disaggregate simplicial decomposition (DSD) algorithm and the gradient projection (GP) algorithm. Application of GP to solve the traffic assignment problem is relatively new, but GP is as good as or better than DSD in direct comparisons.

In this paper, we develop a GP algorithm for the proposed cost-based system optimum model, based on the GoldsteinLevitin-Polyak (GLP) GP method formulated by Bertsekas [30] for general nonlinear multicommodity problems which is successfully used for solving traffic assignment problems [26]. The proposed GLP algorithm includes five key operations: (1) assigning the flow on each path to the links along to find the total link flows, (2) computing link travel times and path travel times and sorting path travel times in decreasing order, (3) computing the first derivative lengths (marginal social path travel costs) for all paths between each OD pair and finding the shortest first derivative lengths for each OD pair, (4) finding the second derivative lengths for each OD pair, and (5) updating the path flows using the second derivative lengths as scaling.

In the next section, a system optimum problem in cost units is formulated in fixed demand networks with continuously distributed value of time. In Section 3, we develop a GP algorithm for the cost-based system optimum model. A numerical example is presented in Section 4 for testing the GP algorithm. Section 5 concludes the paper.

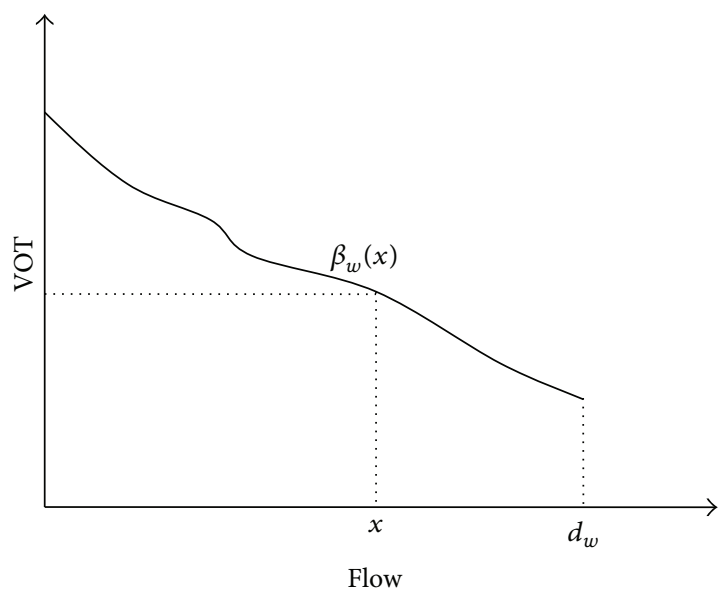

FIGURE 1: Continuously distributed VOTs for users between each OD pair $w$.

\section{Cost-Based System Optimum Problem}

In this section, we formulate the system optimum in cost units in a fixed demand network with heterogeneous users in terms of a continuously distributed VOT. Let $G=(V, A)$ be a directed network where $V$ is the set of nodes and $A$ the set of links. Each link $a \in A$ has an associated flow-dependent travel time $t_{a}\left(v_{a}\right)$ which is assumed to be differentiable, convex, and monotonically increasing subject to the link flow $v_{a}$. Let $W$ be the set of all OD pairs, $R_{w}$ the set of all paths connecting OD pair $w \in W$, and $\left|R_{w}\right|$ the number of paths between each OD pair. Let $S_{w}^{r}$ be the set of drivers (users) on path $r \in R_{w}$ and $f_{w}^{r}$ the number of these drivers; $\mathbf{f}=$ $\left(\ldots, f_{w}^{r}, \ldots\right)$. We have $v_{a}=\sum_{w \in W} \sum_{r \in R_{w}} f_{w}^{r} \delta_{w}^{a r}, a \in A$, where $\delta_{w}^{a r}$ equals 1 if path $r$ between OD pair $w$ contains link $a$, and 0 otherwise. All users on link $a \in A$ are charged by an exogenously given toll $\tau_{a} \geq 0$. Each path $r$ between each OD pair $w$ is associated with a travel time $t_{w}^{r}(\mathbf{f})$ and a travel cost (dollar) $\tau_{w}^{r} \geq 0, t_{w}^{r}(\mathbf{f})=\sum_{a \in A} t_{a}\left(v_{a}\right) \delta_{w}^{a r}$, and $\tau_{w}^{r}=\sum_{a \in A} \tau_{a} \delta_{w}^{a r}$.

Let $d_{w}$ be the travel demand between each OD pair $w$. Instead of assuming a unified VOT for the whole population, in this paper we set a unique, specific VOT for each trip-maker. Let the distribution of VOTs across individuals between each OD pair $w$ be characterized by a continuous function $\beta_{w}(x)>0$. Furthermore, let the population between each OD pair be ordered in decreasing order of their VOTs; that is, $d \beta_{w}(x) / d x \leq 0$, where $x$ is the $x$ th user between each OD pair $w$, as demonstrated in Figure 1. Note that here the VOT distribution is given. In fact, there exist many studies which address estimation of VOTs. By observing choices among alternative combinations of cost and travel time, based on stated preference (SP) analysis, due to the ability to control time and cost variables [31], information about the relative weighting of cost and time can be inferred, and from this the distribution of VOT can be derived. In general, literature on estimation of VOTs refers to two main models: multinomial logit model [32, 33] and mixed logit model [34, 35]. Recently, the mixed logit approach is popular because it does not have 
to assume the irrelevance of independent alternatives (IIA) property.

Let $j=1,2, \ldots,\left|R_{w}\right|$ denote the paths between each OD pair $w$, satisfying $t_{w}^{1}(\mathbf{f}) \geq t_{w}^{2}(\mathbf{f}) \geq \cdots \geq t_{w}^{\left|R_{w}\right|}(\mathbf{f})$. The system optimum naturally requires that the users with higher VOTs should choose faster paths and those with lower VOTs choose slower paths. Otherwise, the total travel cost can be reduced by switching a higher VOT user on a slower path into a faster path. For the sake of notational consistency, we define $\sum_{k=\left|R_{w}\right|+1}^{\left|R_{w}\right|} f_{w}^{k}=0$ and $\sum_{k=1}^{0} f_{w}^{k}=0$. The system optimum in cost units can be formulated as the following minimization problem:

$$
\min _{\mathbf{f}} Z(\mathbf{f})=\sum_{w \in W} \sum_{r=1}^{\left|R_{w}\right|} t_{w}^{r}(\mathbf{f})\left(\int_{0}^{f_{w}^{r}} \beta_{w}\left(\sum_{k=r+1}^{\left|R_{w}\right|} f_{w}^{k}+x\right) d x\right),
$$

subject to

$$
\begin{gathered}
\sum_{k=1}^{\left|R_{w}\right|} f_{w}^{k}=d_{w}, \\
f_{w}^{k} \geq 0,
\end{gathered}
$$

where $t_{w}^{r}(\mathbf{f})=\sum_{a \in w} t_{a}\left(v_{a}\right) \delta_{w}^{a r}, v_{a}=\sum_{w \in W} \sum_{r \in R_{w}} f_{w}^{r} \delta_{w}^{a r}$. Recall that the users between each OD pair are arranged in decreasing order of their VOTs. This determines the integral formulation appearing in (1).

The first-order optimality conditions of the above minimization problem are as follows:

$$
\begin{aligned}
& t_{w}^{r}(\mathbf{f}) \beta_{w}\left(\sum_{k=r}^{\left|R_{w}\right|} f_{w}^{k}\right)+\sum_{w \in W} \sum_{h=1}^{\left|R_{w}\right|} \sum_{a \in A} \frac{d t_{a}\left(v_{a}\right)}{d v_{a}} \delta_{a r}^{w} \delta_{a h}^{w} \\
& \quad \times \int_{0}^{f_{w}^{h}} \beta_{w}\left(\sum_{k=h+1}^{\left|R_{w}\right|} f_{w}^{k}+x\right) d x \\
& \quad+\sum_{k=1}^{r-1} t_{w}^{k}(\mathbf{f}) \int_{0}^{f_{w}^{k}} \frac{\partial \beta_{w}\left(\sum_{h=k+1}^{\left|R_{w}\right|} f_{w}^{h}+x\right)}{\partial f_{w}^{r}} d x=g_{w, \mathrm{cost}}^{\text {so }} \\
& \quad \text { if } f_{w}^{r}>0, \quad r=1,2, \ldots,\left|R_{w}\right|, \quad w \in W, \\
& \quad \times \int_{0}^{r}(\mathbf{f}) \beta_{w}\left(\sum_{k=r}^{\left|R_{w}\right|} f_{w}^{k}\right)+\sum_{w \in W} \sum_{h=1}^{f_{w}^{h}} \sum_{a \in A} \frac{d t_{a}\left(v_{a}\right)}{d v_{a}} \delta_{a r}^{w} \delta_{a h}^{w} \\
& \quad+\sum_{k=h+1}^{\left|R_{w}\right|} t_{w}^{k-1}(\mathbf{f}) \int_{0}^{f_{w}^{k}} \frac{\partial \beta_{w}\left(\sum_{h=k+1}^{\left|R_{w}\right|} f_{w}^{h}+x\right)}{\partial f_{w}^{r}} d x \geq g_{w, \operatorname{cost}}^{\mathrm{so}} \\
& \quad \text { if } f_{w}^{r}=0, \quad r=1,2, \ldots,\left|R_{w}\right|, \quad w \in W,
\end{aligned}
$$

where $g_{w, \text { cost }}^{\text {so }}$ is the minimal travel cost between each OD pair $w$.
Note that the sum of the second and third terms on the left hand side of (4) is the total externality caused by the $x$ th user of OD pair $w, x=\sum_{k=r}^{\left|R_{w}\right|} f_{w}^{k}$, or the $f_{w}^{r}$ th user of path $r$. The second term states that this new trip-maker imposes additional costs on all users who may belong to other OD pairs and other paths and have their own VOTs but traverse the links of path $r$. This is the traditional congestion externality reported in the literature. The third term states that the costs of paths 1 to $r-1$ are reduced since the $f_{w}^{r}$ th user's path choice changes the VOTs of the users on these paths (this user chooses path $r$ rather than other longer paths). This is another kind of externality attributed to the VOT distribution of OD trips. Let

$$
\begin{aligned}
c_{w}^{r}(\mathbf{f})= & t_{w}^{r}(\mathbf{f}) \beta_{w}\left(\sum_{k=r}^{\left|R_{w}\right|} f_{w}^{k}\right)+\sum_{w \in W} \sum_{h=1}^{\left|R_{w}\right|} \sum_{a \in A} \frac{d t_{a}\left(v_{a}\right)}{d v_{a}} \delta_{a r}^{w} \delta_{a h}^{w} \\
& \times \int_{0}^{f_{w}^{h}} \beta_{w}\left(\sum_{k=h+1}^{\left|R_{w}\right|} f_{w}^{k}+x\right) d x \\
& +\sum_{k=1}^{r-1} t_{w}^{k}(\mathbf{f}) \int_{0}^{f_{w}^{k}} \frac{\partial \beta_{w}\left(\sum_{h=k+1}^{\left|R_{w}\right|} f_{w}^{h}+x\right)}{\partial f_{w}^{r}} d x .
\end{aligned}
$$

Also, for the remainder of the paper when we refer to the first derivative lengths we mean the first derivatives of the objective function, which can also be regarded as marginal social travel cost corresponding to path flow on path $r$.

Equations (4) and (5) can then be rewritten as

$$
\begin{array}{r}
c_{w}^{r}(\mathbf{f})=g_{w, \text { cost }}^{\text {so }}, \quad \text { if } f_{w}^{r}>0, r=\left\{1,2, \ldots,\left(\left|R_{w}\right|-1\right)\right\}, \\
w \in W, \\
c_{w}^{r}(\mathbf{f}) \geq g_{w, \text { cost }}^{\text {so }}, \quad \text { if } f_{w}^{r}=0, r=\left\{1,2, \ldots,\left(\left|R_{w}\right|-1\right)\right\}, \\
w \in W .
\end{array}
$$

Therefore, in a network where each user has a unique VOT, (7) state that, at optimality, the marginal social travel costs on all the used paths connecting a given OD pair are equal and less than or equal to those on all the unused paths. This is similar to a standard system optimum, in which, at optimality, the marginal social travel times on all the used paths between each given OD pair are equal and less than or equal to those on all the unused paths.

Clearly, the proposed model is more complicated than a standard system optimum. According to the extreme value theorem that a continuous function in the closed and bounded space attains its maximum and minimum, the above minimization program must attain its minimum value. The most common algorithm used to solve traffic assignment problems is link-based Frank-Wolfe algorithm introduced by LeBlanc et al. [36]. However, the objective function is, in general, not convex since the path travel costs depending not only on its own path but also on other paths are inseparable and asymmetric in terms of the path flows. Fortunately, for 
the differentiable and bounded objective, the convexity of the constraints allows the development of a gradient projection algorithm.

\section{Path-Based Traffic Assignment Algorithm}

In this section, we adopt the Goldstein-Levitin-Polyak algorithm to the traffic assignment problem. In each iteration, the travel demand constraints (2) are eliminated by reformulating the path-flow variables in terms of nonshortest path flows in terms of the first derivative lengths to make projection operation simpler. This is implemented by partitioning $f_{w}^{r}$ into the shortest path flow $f_{w}^{\bar{r}}$ and the nonshortest path flows $f_{w}^{r}$ and writing constraints (2) in terms of $f_{w}^{r}$ as follows:

$$
f_{w}^{\bar{r}}=d_{w}-\sum_{r=1, r \neq \bar{r}}^{\left|R_{w}\right|} f_{w}^{r}, \quad \forall w \in W .
$$

It should be noted that $\bar{r}$ is the shortest path in terms of the first derivative lengths but $\left|R_{w}\right|$ is the shortest path in terms of path travel times. Putting constraints (8) into the objective function, we obtain a new formulation with just the nonnegativity constraints on the nonshortest path flows as the decision variables. Consider

$$
\begin{gathered}
\min \widetilde{Z}(\widetilde{\mathbf{f}}) \\
f_{w}^{r} \geq 0, \quad \forall r \in R_{w}, \quad r \neq \bar{r}, w \in W,
\end{gathered}
$$

where $\widetilde{Z}(\widetilde{\mathbf{f}})$ is the reformulated objective function only including the nonshortest path flows for all OD pairs. This reformulation is a program with only nonnegativity constraints. The first and second derivatives of the new objective function can be easily derived as follows:

$$
\begin{array}{r}
\frac{\partial Z(\widetilde{\mathbf{f}})}{\partial f_{w}^{r}}=\frac{\partial Z(\mathbf{f})}{\partial f_{w}^{r}}-\frac{\partial(\mathbf{f})}{\partial f_{w}^{\bar{r}}}=c_{w}^{r}(\mathbf{f})-c_{w}^{\left|R_{w}\right|}(\mathbf{f}), \\
\forall r \in R_{w}, \quad r \neq \bar{r}, \quad w \in W, \\
\frac{\partial^{2} Z(\widetilde{\mathbf{f}})}{\left(\partial f_{w}^{r}\right)^{2}}=\frac{\partial^{2} Z(\mathbf{f})}{\left(\partial f_{w}^{r}\right)^{2}}-2 \frac{\partial^{2} Z(\mathbf{f})}{\partial f_{w}^{r} \partial f_{w}^{\bar{r}}}+\frac{\partial^{2} Z(\mathbf{f})}{\left(\partial f_{w}^{\bar{r}}\right)^{2}},
\end{array}
$$$$
\forall r \in R_{w}, \quad r \neq\left|R_{w}\right|, \quad w \in W,
$$

where

$$
\begin{aligned}
\frac{\partial^{2} Z(\mathbf{f})}{\left(\partial f_{w}^{r}\right)^{2}}= & \frac{\partial \beta_{w}\left(\sum_{k=r}^{\left|R_{w}\right|} f_{w}^{k}\right)}{\partial f_{w}^{r}} \sum_{a \in A} t_{a}\left(v_{a}\right) \delta_{a r}^{w} \\
& +2 \beta_{w}\left(\sum_{k=r}^{\left|R_{w}\right|} f_{w}^{k}\right) \sum_{a \in A} \frac{d t_{a}\left(v_{a}\right)}{d v_{a}} \delta_{a r}^{w} \\
& +\sum_{w \in W h=1} \sum_{a \in A}^{\left|R_{w}\right|} \frac{d^{2} t_{a}\left(v_{a}\right)}{\left(d v_{a}\right)^{2}} \delta_{a r}^{w} \delta_{a h}^{w} \\
& \times \int_{0}^{f_{w}^{h}} \beta_{w}\left(\sum_{k=h+1}^{\left|R_{w}\right|} f_{w}^{k}+x\right) d x
\end{aligned}
$$

$$
\begin{aligned}
& +2 \sum_{h=1}^{r-1} \sum_{a \in A} \frac{d t_{a}\left(v_{a}\right)}{d v_{a}} \delta_{a r}^{w} \delta_{a h}^{w} \\
& \times \int_{0}^{f_{w}^{h}} \frac{\partial \beta_{w}\left(\sum_{k=h+1}^{\left|R_{w}\right|} f_{w}^{k}+x\right)}{\partial f_{w}^{r}} d x \\
& +\sum_{k=1}^{r-1} t_{w}^{k}(\mathbf{f}) \int_{0}^{f_{w}^{k}} \frac{\partial^{2} \beta_{w}\left(\sum_{h=k+1}^{\left|R_{w}\right|} f_{w}^{h}+x\right)}{\left(\partial f_{w}^{r}\right)^{2}} d x, \\
& \frac{\partial^{2} Z(\mathbf{f})}{\partial f_{w}^{r} \partial f_{w}^{\bar{r}}}=\frac{\partial \beta_{w}\left(\sum_{k=r}^{\left|R_{w}\right|} f_{w}^{k}\right)}{\partial f_{w}^{\bar{r}}} \sum_{a \in A} t_{a}\left(v_{a}\right) \delta_{a r}^{w} \\
& +\beta_{w}\left(\sum_{k=\bar{r}}^{\left|R_{w}\right|} f_{w}^{k}\right) \sum_{a \in A} \frac{d t_{a}\left(v_{a}\right)}{d v_{a}} \delta_{a r}^{w} \delta_{a \bar{r}}^{w} \\
& +\beta_{w}\left(\sum_{k=r}^{\left|R_{w}\right|} f_{w}^{k}\right) \sum_{a \in A} \frac{d t_{a}\left(v_{a}\right)}{d v_{a}} \delta_{a r}^{w} \delta_{a \bar{r}}^{w} \\
& +\sum_{w \in W} \sum_{h=1}^{\left|R_{w}\right|} \sum_{a \in A} \frac{d^{2} t_{a}\left(v_{a}\right)}{\left(d v_{a}\right)^{2}} \delta_{a r}^{w} \delta_{a \bar{r}}^{w} \delta_{a h}^{w} \\
& \times \int_{0}^{f_{w}^{h}} \beta_{w}\left(\sum_{k=h+1}^{\left|R_{w}\right|} f_{w}^{k}+x\right) d x \\
& +\sum_{h=1}^{\bar{r}-1} \sum_{a \in A} \frac{d t_{a}\left(v_{a}\right)}{d v_{a}} \delta_{a r}^{w} \delta_{a h}^{w} \\
& \times \int_{0}^{f_{w}^{h}} \frac{\partial \beta_{w}\left(\sum_{k=h+1}^{\left|R_{w}\right|} f_{w}^{k}+x\right)}{\partial f_{w}^{\bar{r}}} d x \\
& +\sum_{k=1}^{r-1} \sum_{a \in A} \frac{d t_{a}\left(v_{a}\right)}{d v_{a}} \delta_{a k}^{w} \delta_{a \bar{r}}^{w} \\
& \times \int_{0}^{f_{w}^{k}} \frac{\partial \beta_{w}\left(\sum_{h=k+1}^{\left|R_{w}\right|} f_{w}^{h}+x\right)}{\partial f_{w}^{r}} d x \\
& +\sum_{k=1}^{r-1} t_{w}^{k} \int_{0}^{f_{w}^{k}} \frac{\partial^{2} \beta_{w}\left(\sum_{h=k+1}^{\left|R_{w}\right|} f_{w}^{h}+x\right)}{\partial f_{w}^{r} \partial f_{w}^{\bar{r}}} d x, \\
& \frac{\partial^{2} Z(\mathbf{f})}{\left(\partial f_{w}^{\bar{r}}\right)^{2}}=\frac{\partial \beta_{w}\left(\sum_{k=\bar{r}}^{\left|R_{w}\right|} f_{w}^{k}\right)}{\partial f_{w}^{\bar{r}}} \sum_{a \in A} t_{a}\left(v_{a}\right) \delta_{a \bar{r}}^{w} \\
& +2 \beta_{w}\left(\sum_{k=\bar{r}}^{\left|R_{w}\right|} f_{w}^{k}\right) \sum_{a \in A} \frac{d t_{a}\left(v_{a}\right)}{d v_{a}} \delta_{a \bar{r}}^{w} \\
& \times \sum_{w \in W} \sum_{h=1}^{\left|R_{w}\right|} \sum_{a \in A} \frac{d^{2} t_{a}\left(v_{a}\right)}{\left(d v_{a}\right)^{2}} \delta_{a \bar{r}}^{w} \delta_{a h}^{w} \\
& \times \int_{0}^{f_{w}^{h}} \beta_{w}\left(\sum_{k=h+1}^{\left|R_{w}\right|} f_{w}^{k}+x\right) d x \\
& +2 \sum_{h=1}^{\bar{r}-1} \sum_{a \in A} \frac{d t_{a}\left(v_{a}\right)}{d v_{a}} \delta_{a \bar{r}}^{w} \delta_{a h}^{w}
\end{aligned}
$$




$$
\begin{aligned}
& \times \int_{0}^{f_{w}^{h}} \frac{\partial \beta_{w}\left(\sum_{k=h+1}^{\left|R_{w}\right|} f_{w}^{k}+x\right)}{\partial f_{w}^{\bar{r}}} d x \\
& +\sum_{k=1}^{\bar{r}-1} t_{w}^{k}(\mathbf{f}) \int_{0}^{f_{w}^{k}} \frac{\partial^{2} \beta_{w}\left(\sum_{h=k+1}^{\left|R_{w}\right|} f_{w}^{h}+x\right)}{\left(\partial f_{w}^{\bar{r}}\right)^{2}} d x .
\end{aligned}
$$

Putting (13) into (12) yields

$$
\begin{aligned}
& \frac{\partial^{2} Z(\widetilde{\mathbf{f}})}{\left(\partial f_{w}^{r}\right)^{2}}=\frac{\partial \beta_{w}\left(\sum_{k=r}^{\left|R_{w}\right|} \bar{f}_{w}^{k}\right)}{\partial f_{w}^{r}} \sum_{a \in A}\left(t_{w}^{\bar{r}}(\mathbf{f})-t_{w}^{r}(\mathbf{f})\right) \\
& +2 \beta_{w}\left(\sum_{k=r}^{\left|R_{w}\right|} f_{w}^{k}\right) \sum_{a \in A} \frac{d t_{a}\left(v_{a}\right)}{d v_{a}} \delta_{a r}^{w}\left(1-\delta_{a \bar{r}}^{w}\right) \\
& +2 \beta_{w}\left(\sum_{k=\bar{r}}^{\left|R_{w}\right|} \bar{f}_{w}^{k}\right) \sum_{a \in A} \frac{d t_{a}\left(v_{a}\right)}{d v_{a}} \delta_{a \bar{r}}^{w}\left(1-\delta_{a r}^{w}\right) \\
& +\sum_{w \in W h=1} \sum_{a \in A}^{\left|R_{w}\right|} \sum_{a} \frac{d^{2} t_{a}\left(v_{a}\right)}{\left(d v_{a}\right)^{2}}\left(\delta_{a r}^{w}-\delta_{a \bar{r}}^{w}\right)^{2} \delta_{a h}^{w} \\
& \times \int_{0}^{f_{w}^{h}} \beta_{w}\left(\sum_{k=h+1}^{\left|R_{w}\right|} f_{w}^{k}+x\right) d x \\
& +2 \sum_{h=1}^{r-1} \sum_{a \in A} \frac{d t_{a}\left(v_{a}\right)}{d v_{a}}\left(\delta_{a r}^{w}-\delta_{a \bar{r}}^{w}\right) \delta_{a h}^{w} \\
& \times \int_{0}^{f_{w}^{h}} \frac{\partial \beta_{w}\left(\sum_{k=h+1}^{\left|R_{w}\right|} f_{w}^{k}+x\right)}{\partial f_{w}^{r}} d x \\
& +2 \sum_{h=1}^{\bar{r}-1} \sum_{a \in A} \frac{d t_{a}\left(v_{a}\right)}{d v_{a}}\left(\delta_{a \bar{r}}^{w}-\delta_{a r}^{w}\right) \delta_{a h}^{w} \\
& \times \int_{0}^{f_{w}^{h}} \frac{\partial \beta_{w}\left(\sum_{k=h+1}^{\left|R_{w}\right|} f_{w}^{k}+x\right)}{\partial f_{w}^{\bar{r}}} d x \\
& +\sum_{k=r}^{\left|R_{w}\right|-1} t_{w}^{k} \int_{0}^{f_{w}^{k}} \frac{\partial^{2} \beta_{w}\left(\sum_{h=k+1}^{\left|R_{w}\right|} f_{w}^{h}+x\right)}{\left(\partial f_{w}^{\bar{r}}\right)^{2}} d x, \\
& \forall r \in R_{w}, \quad r \neq \bar{r}, \quad w \in W .
\end{aligned}
$$

Note that the second derivative is, in general, not positive. This is different from the transformed objective function of the standard system optimum model [25]. Thus, in each iteration, the scaled GLP algorithm updates the path flows according to the following iteration equations:

$$
\begin{array}{r}
f_{w}^{r}(n+1)=\left[f_{w}^{r}(n)-\frac{\alpha(n)}{s_{w}^{r}(n)}\left(c_{w}^{r}(\mathbf{f}(n))-c_{w}^{\bar{k}_{w}(n)}(\mathbf{f}(n))\right]^{+},\right. \\
\quad \text { if } s_{w}^{r}(n)>0, \quad \forall r \in R_{w}, \quad r \neq \bar{k}_{w}(n), \quad w \in W,
\end{array}
$$

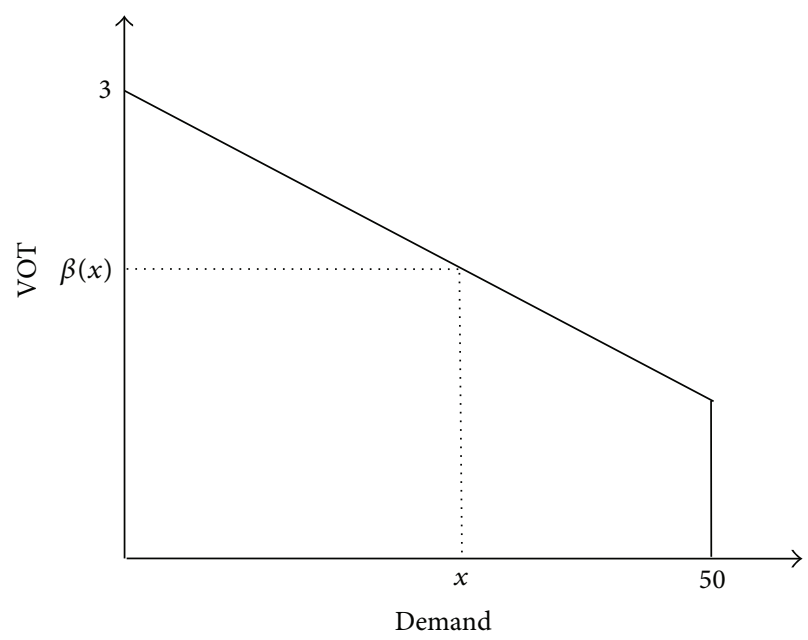

FIGURE 2: Continuously distributed VOTs of users.

$$
\begin{gathered}
f_{w}^{r}(n+1)=f_{w}^{r}(n), \quad \text { if } s_{w}^{r}(n) \leq 0, \forall r \in R_{w}, \quad r \neq \bar{k}_{w}(n), \\
w \in W, \\
f_{w}^{\bar{k}_{w}(n)}(n+1)=d_{w}-\sum_{r=1, r \neq \bar{k}_{w}(n)}^{\left|R_{w}\right|} f_{w}^{r}(n+1),
\end{gathered}
$$

where $n$ is the iteration number, $\alpha(n)$ is the step size, $\bar{k}_{w}(n)$ is the shortest path in terms of the first derivative lengths between each OD pair $w, s_{w}^{r}(n)$ is a diagonal scaling, equivalent to the second derivative regarding path $r$, that is, $s_{w}^{r}(n)=\partial^{2} Z(\widetilde{\mathbf{f}}) /\left(\partial f_{w}^{r}\right)^{2}, c_{w}^{r}(\mathbf{f}(n))$ and $c_{w}^{\bar{k}_{w}(n)}(\mathbf{f}(n))$ are the first derivative lengths along path $r$ and path $\bar{k}_{w}(n)$ between each OD pair $w$, and []$^{+}$denotes the projection operation.

Note that $c_{w}^{r}(\mathbf{f}(n))-c_{w}^{\bar{k}_{w}(n)}(\mathbf{f}(n))$ can be explained by (11), and (15) certainly satisfies the flow conservation constraints in (2) and nonnegativity conditions in (3).

With the above flow update equations, the complete algorithmic steps can be summarized as follows.

Step 1 (initialization). Set $t_{a}(0)$, for all $a$, and perform all-ornothing assignments. This yields path flows $\mathbf{f}^{w}(1)$, for all $w \in$ $W$, and link flows $x_{a}(1)$, for all $a$. Set iteration counter $n=l$. Initialize the path-set $R_{w}$ with the shortest path for each OD pair $w$.

Step 2 (update). Set $t_{a}(n)=t_{a}\left(x_{a}(n)\right)$, for all $a$. Sort path travel time in decreasing order; that is, $t_{w}^{1}(n) \geq t_{w}^{2}(n) \geq \cdots \geq$ $t_{w}^{\left|R_{w}\right|}(n)$, where $t_{w}^{k}(n)=\sum_{a \in A} t_{a}\left(x_{a}(n)\right) \delta_{a k}^{w}, k=1,2, \ldots,\left|R_{w}\right|$.

Step 3. Update the first derivative lengths $c_{w}^{k}(n)$ of all the paths $k$ in $R_{w}$, for all $w \in W$.

Step 4 (direction finding). Find the shortest paths $\bar{k}_{w}(n)$ in terms of marginal social travel cost between each OD pair $w$, based on $\left\{c_{w}^{k}(n)\right\}$. If $\bar{k}_{w}(n) \notin R_{w}$, then add it to $R_{w}$ and record 


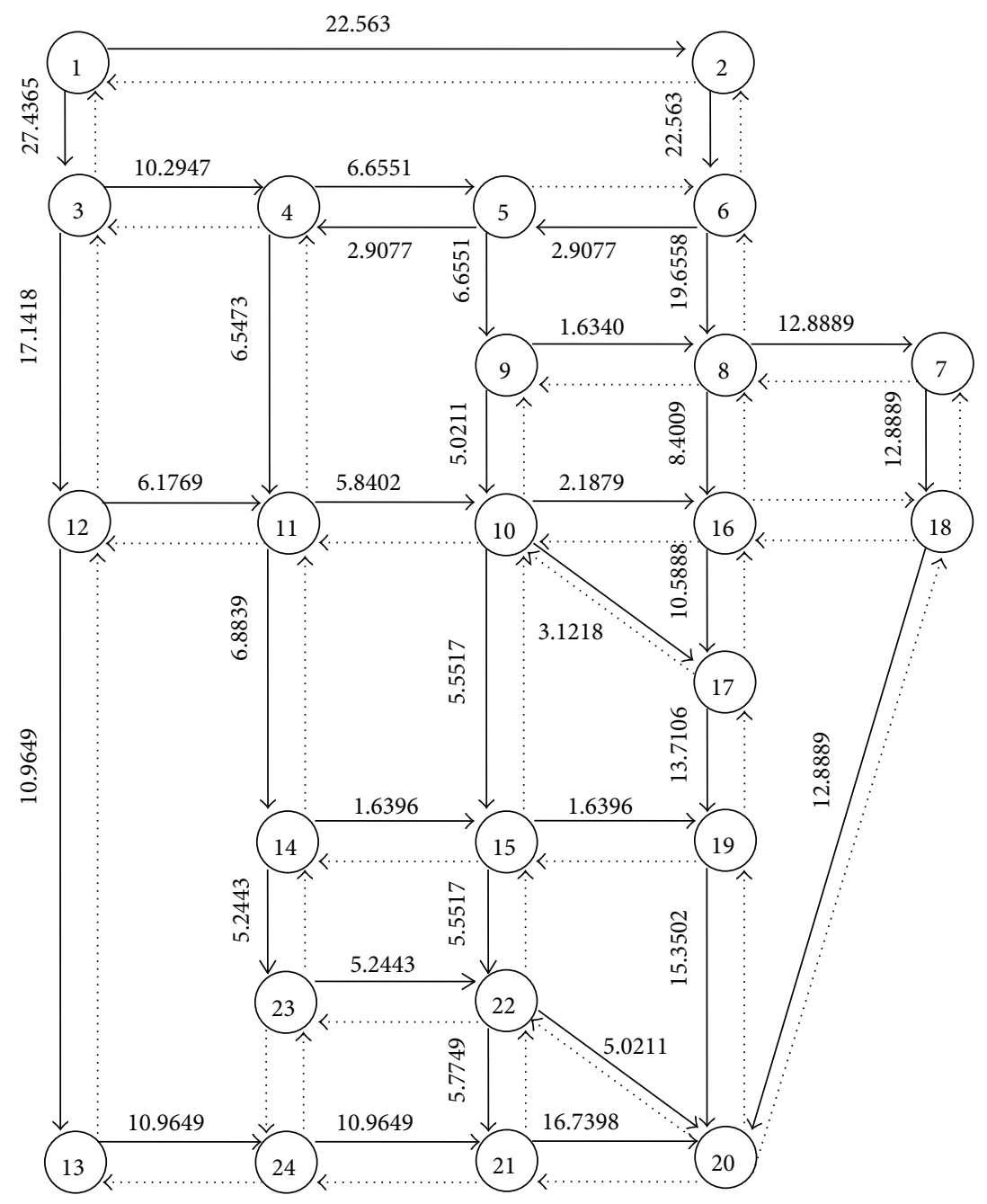

Figure 3: The Sioux Falls test network.

$c_{w}^{\bar{k}_{w}^{n}}(n)$. Otherwise, tag the shortest among the paths in $R_{w}$ as $\bar{k}_{w}(n)$.

Step 5 (move). Set the new path flows as follows:

$$
\begin{array}{r}
f_{w}^{k}(n+1)=\max \left\{0, f_{w}^{k}(n)-\frac{\alpha(n)}{s_{w}^{k}(n)}\left(c_{w}^{k}(n)-c_{w}^{\bar{k}_{w}(n)}(n)\right)\right\}, \\
\text { if } s_{w}^{r}(n)>0, \quad \forall k \in R_{w}, \quad k \neq \bar{k}_{w}(n), \quad w \in W, \\
f_{w}^{k}(n+1)=f_{w}^{k}(n), \quad \text { if } s_{w}^{r}(n) \leq 0, \quad \forall k \in R_{w}, \quad k \neq \bar{k}_{w}(n), \\
w \in W,
\end{array}
$$

where $s_{w}^{k}(n)=\partial^{2} Z(\tilde{f}) /\left(\partial f_{w}^{k}\right)^{2}$, for all $k \in R_{w}$, and $\alpha(n)$ is a scalar step-size modifier.

Also, $f_{w}^{\bar{k}_{w}(n)}(n+1)=d_{w}-\sum_{r=1, r \neq \bar{k}_{w}(n)}^{\left|R_{w}\right|} f_{w}^{r}(n+1)$, for all $k \in R_{w}, k \neq \bar{k}_{w}(n)$.

Update link flows $x_{a}(n+1)=\sum_{w \in W} \sum_{k \in R_{w}} f_{w}^{k}(n+1) \delta_{k a}^{w}$.
Step 6 (convergence test). Determine the total deviation of marginal social travel costs between all OD pairs $E=$ $\sum_{w \in W} \sum_{k \in R_{w}}\left(f_{w}^{k}(n) / d_{w}\right) \cdot\left|\left(c_{w}^{k}(n)-c_{w}^{\bar{k}_{w}(n)}(n)\right) / c_{w}^{k}(n)\right|$. If $E \leq \varepsilon$, then stop. Otherwise, set $n=n+l$ and go to Step 2 .

For convenience, it is better to keep $\alpha(n)$ constant (i.e., $\alpha(n)=\alpha$ ) since $s_{w}^{k}(n)$ is used for scaling [26]. Given any starting set of path flows, there exists $\alpha$ such that if $\alpha \in[0, \bar{\alpha}]$ the sequence generated by this algorithm converges to the objective function (1) [37].

\section{Numerical Example}

In the section, a numerical example is presented to illustrate the effectiveness of the proposed model and algorithm. The test network is the Sioux Falls network, which is a medium sized network with 24 nodes and 76 links, as shown in Figure 3. This paper considers only one origin-destination pair from node 1 to node 20 for conveniently providing a complete picture of the travel pattern which demonstrates all used 
TABLE 1: Generated paths, path costs, and VOTs.

\begin{tabular}{lcccc}
\hline Paths & Link set & Path flow & Path cost & VOT \\
\hline Path 1 & $1-2-6-5-4-11-14-23-22-21-20$ & 2.9481 & 166.7692 & {$[1.0000,1.1179]$} \\
Path 2 & $1-2-6-8-7-18-20$ & 12.8851 & 163.4423 & {$[1.1179,1.9039]$} \\
Path 3 & $1-2-6-8-16-17-19-20$ & 6.7645 & 151.2485 & {$[1.6333,1.9039]$} \\
Path 4 & $1-3-4-11-14-23-22-21-20$ & 0.9821 & 124.8157 & {$[1.9039,1.9432]$} \\
Path 5 & $1-3-4-5-9-8-16-17-19-20$ & 1.6310 & 122.9879 & {$[1.9432,2.0084]$} \\
Path 6 & $1-3-4-11-10-17-19-20$ & 2.6448 & 122.7562 & {$[2.0084,2.1142]$} \\
Path 7 & $1-3-12-11-14-23-22-21-20$ & 1.3288 & 121.6256 & {$[2.1142,2.1674]$} \\
Path 8 & $1-3-4-5-9-10-15-22-20$ & 5.0010 & 121.1957 & {$[2.1674,2.3674]$} \\
Path 9 & $1-3-12-11-14-15-19-20$ & 1.6379 & 120.9318 & {$[2.3674,2.4329]$} \\
Path 10 & $1-3-12-11-10-16-17-19-20$ & 2.1797 & 120.5415 & {$[2.4329,2.5201]$} \\
Path 11 & $1-3-12-11-10-17-19-20$ & 0.4598 & 119.5662 & {$[2.5201,2.5385]$} \\
Path 12 & $1-3-12-11-10-15-22-21-20$ & 0.5293 & 119.2367 & {$[2.5385,2.5597]$} \\
Path 13 & $1-3-12-13-24-21-20$ & 11.0078 & 117.8441 & {$[2.5597,3.0000]$} \\
\hline
\end{tabular}

path flows, all used link flows, and path choice behaviors. We here use the standard Bureau of Public Road (BPR) link cost function for our numerical study. The functional form is given by

$$
t_{a}\left(x_{a}\right)=t_{f}\left(1+0.15\left(\frac{x_{a}}{C_{a}}\right)^{4}\right),
$$

where $t_{f}$ is the free-flow cost, $x_{a}$ is the flow, and $C_{a}$ is the link capacity.

Assume that the total demand $d_{12}$ is 50 , and all network users which are differed by a continuously distributed VOT are given in Figure 2 as follows:

$$
\beta(x)=3-\frac{x}{25}, \quad x \in[0,50] .
$$

Note that, in the numerical example, we show that $\alpha=1$ achieves very good convergence rate.

The GP algorithm provides a complete picture of the travel pattern and keeps track of the distribution of the OD flows among the different routes, as shown in Table 1 and Figure 3. In the Sioux Falls network, there are many paths between OD pair 1-20. However, the number of the used paths to define the system optimum keeps small; that is, there are only thirteen used paths which are given in a decreasing order in terms of path travel times. It can be seen that, at optimality, people with high VOTs would choose faster paths, whereas people with low VOTs would choose slower paths. This is consistent with the requirements for the system optimum in Section 2.

Figure 3 shows the optimal link-flow distribution. Note that the real lines refer to the used links and the dotted lines refer to the unused paths. The link flows equal the values beside the corresponding links and are also graphically demonstrated by the widths of the corresponding lines. It can be easily seen that in the network most links in the forward directions are used, but most links in the backward directions are unused.

Table 2 compares the total travel times and total travel costs under different types of traffic assignment, respectively.
TABLE 2: Total travel times and total travel costs under different types of traffic assignment.

\begin{tabular}{lcc}
\hline Type of traffic assignment & $\begin{array}{c}\text { Total travel } \\
\text { time }\end{array}$ & $\begin{array}{c}\text { Total travel } \\
\text { cost }\end{array}$ \\
\hline User equilibrium & 6827 & 13655 \\
Time-based system optimum & 6723 & 13343 \\
Cost-based system optimum & 6912 & 13281 \\
\hline
\end{tabular}

At the cost-based system optimum, the total travel cost is the lowest whereas the total travel time is the highest. At the timebased system optimum, the total travel time is the lowest, and the total travel cost is more than the cost-based system optimum but less than the user equilibrium.

Figure 4 depicts the pattern of convergence toward the minimum. This convergence pattern is demonstrated in terms of the reduction in the value of the objective function from iteration to iteration. After the 21st iteration, the marginal contribution of each successive iteration becomes smaller and smaller as the algorithm proceeds (this property is the basis for the convergence criterion used in the example). After the 40th iteration, the value of the objective function almost keeps unchanged and is approximately equal to the minimum value 13281, verifying the effectiveness of the proposed GP algorithm.

\section{Conclusions}

The VOT varies significantly across individuals because of the different socioeconomic characteristics, trip purposes, attitudes, and inherent preferences. Furthermore, the VOT of each user is assumed to be deterministic and constant because the factors influencing VOT keep unchanged within a certain time period. We provide a theoretical investigation of the system optimum problem in fixed demand networks with continuous VOT distribution. This system optimum problem is formulated as a minimization program in cost units, which is more complicated than standard system optimum. This is because its objective function is, in general, nonconvex since 


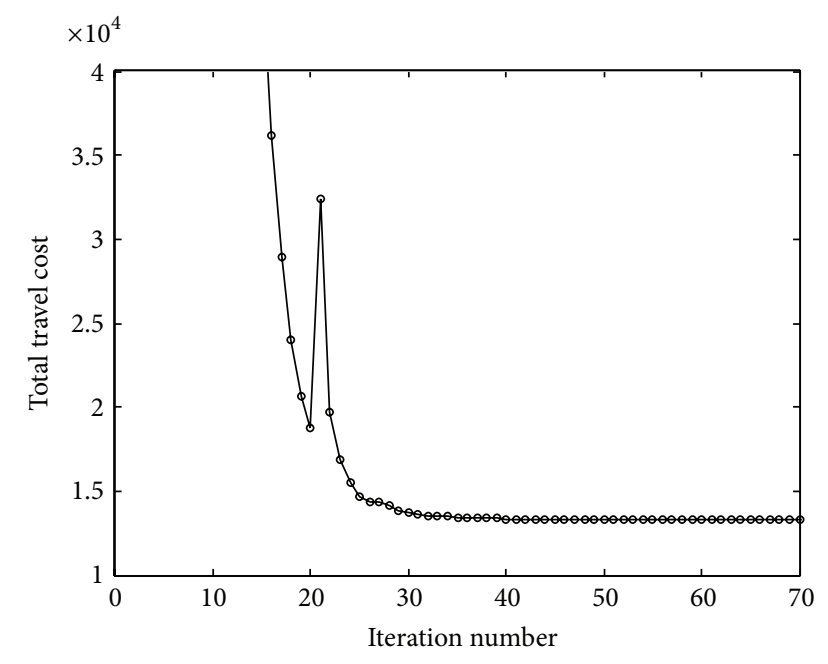

FIGURE 4: The objective function values versus the iterations of the GP algorithm.

path travel costs depending not only on its own path but also on other paths are inseparable and asymmetric in terms of the path flows. Considering the convexity of the constraints, we have developed a path-based GLP algorithm for solving this model. The test results in the Sioux Falls network show the effectiveness of the algorithm.

This study lays a solid foundation for road pricing to realize the cost-based system optimum in fixed demand general networks with heterogeneous users. For future research, this framework can be extended to the case of elastic demand and further investigate how to determine anonymous tolls to realize the system optimum in cost units.

\section{Conflict of Interests}

The authors declare that there is no conflict of interests regarding the publication of this paper.

\section{Acknowledgments}

This research was supported by grants from the National Basic Research Program of China (2012CB725401), the National Natural Science Foundation of China (71071004 and 71271004), and the National High Technology Research and Development Program of China (863 Program) (2012AA112401).

\section{References}

[1] J. G. Wardrop, "Some theoretical aspects of road traffic research," Proceedings of the Institute of Civil Engineers II, no. 1, pp. 325-378, 1952.

[2] H. Yang and H.-J. Huang, "The multi-class, multi-criteria traffic network equilibrium and systems optimum problem," Transportation Research B, vol. 38, no. 1, pp. 1-15, 2004.

[3] K. A. Small and J. Yan, "The value of "value pricing" of roads: second-best pricing and product differentiation," Journal of Urban Economics, vol. 49, no. 2, pp. 310-336, 2001.
[4] D. Brownstone and K. A. Small, "Valuing time and reliability: assessing the evidence from road pricing demonstrations," Transportation Research A, vol. 39, no. 4, pp. 279-293, 2005.

[5] K. A. Small, C. Winston, and J. Yan, "Uncovering the distribution of motorists' preferences for travel time and reliability," Econometrica, vol. 73, no. 4, pp. 1367-1382, 2005.

[6] C. Cirillo and K. W. Axhausen, "Evidence on the distribution of values of travel time savings from a six-week diary," Transportation Research A, vol. 40, no. 5, pp. 444-457, 2006.

[7] C.-C. Lu, H. S. Mahmassani, and X. Zhou, "A bi-criterion dynamic user equilibrium traffic assignment model and solution algorithm for evaluating dynamic road pricing strategies," Transportation Research C, vol. 16, no. 4, pp. 371-389, 2008.

[8] R. Lindsey, "Existence, uniqueness, and trip cost function properties of user equilibrium in the bottleneck model with multiple user classes," Transportation Science, vol. 38, no. 3, pp. 293-314, 2004.

[9] D. Han and H. Yang, "The multi-class, multi-criterion traffic equilibrium and the efficiency of congestion pricing," Transportation Research E, vol. 44, no. 5, pp. 753-773, 2008.

[10] A. Clark, A. Sumalee, S. Shepherd, and R. Connors, "On the existence and uniqueness of first best tolls in networks with multiple user classes and elastic demand," Transportmetrica, vol. 5, no. 2, pp. 141-157, 2009.

[11] T. C. Lam and K. A. Small, "The value of time and reliability: measurement from a value pricing experiment," Transportation Research E, vol. 37, no. 2-3, pp. 231-251, 2001.

[12] E. T. Verhoef and K. A. Small, "Product differentiation on roads: constrained congestion pricing with heterogeneous users," Journal of Transport Economics and Policy, vol. 38, no. 1, pp. 127-156, 2004.

[13] P. Marcotte and D. L. Zhu, "Existence and computation of optimal tolls in multiclass network equilibrium problems," Operations Research Letters, vol. 37, no. 3, pp. 211-214, 2009.

[14] V. van den Berg and E. T. Verhoef, "Congestion tolling in the bottleneck model with heterogeneous values of time," Transportation Research B, vol. 45, no. 1, pp. 60-78, 2011.

[15] D. Zhu, C. Li, and G. Chen, "Existence of strongly valid tolls for multiclass network equilibrium problems," Acta Mathematica Scientia B, vol. 32, no. 3, pp. 1093-1101, 2012.

[16] J. Mayet and M. Hansen, "Congestion pricing with continuously distributed values of time," Journal of Transport Economics and Policy, vol. 34, no. 3, pp. 359-369, 2000.

[17] F. Xiao and H. Yang, "Efficiency loss of private road with continuously distributed value-of-time," Transportmetrica, vol. 4, no. 1, pp. 19-32, 2008.

[18] F. Xiao and H. M. Zhang, "Pareto-improving and selfsustainable pricing for the morning commute with nonidentical commuters," Transportation Science, pp. 1-11, 2013.

[19] L. J. Tian, H. J. Huang, and H. Yang, "Tradable credit schemes for managing bottleneck congestion and modal split with heterogeneous users," Transportation Research E, vol. 54, pp. 113, 2013.

[20] R. Cole, Y. Dodis, and T. Roughgarden, "Pricing network edges for heterogeneous selfish users," in Proceedings of the 35 Annual ACM Symposium on Theory of Computing, pp. 521-530, ACM, San Diego, Calif, USA, 2003.

[21] L. Fleischer, K. Jain, and M. Mahdian, “Tolls for heterogeneous selfish users in multicommodity networks and generalized congestion games," in Proceedings of the 45th Annual IEEE Symposium on Foundations of Computer Science (FOCS '04), pp. 277-285, Rome, Italy, October 2004. 
[22] G. Karakostas and S. G. Kolliopoulos, "Edge pricing of multicommodity networks for heterogeneous selfish users," in Proceedings of the 45th Annual IEEE Symposium on Foundations of Computer Science (FOCS '04), pp. 268-276, Rome, Italy, October 2004.

[23] W. X. Wu and H. J. Huang, "Finding anonymous tolls to realize target flow pattern in networks with continuously distributed value of time," submitted to Transportation Research B, 2013.

[24] R. B. Dial, "Network-optimized road pricing: part II: algorithms and examples," Operations Research, vol. 47, no. 2, pp. 327-336, 1999.

[25] A. Chen, D.-H. Lee, and R. Jayakrishnan, "Computational study of state-of-the-art path-based traffic assignment algorithms," Mathematics and Computers in Simulation, vol. 59, no. 6, pp. 509-518, 2002.

[26] R. Jayakrishnan, W. K. Tsai, J. N. Prashker, and S. Rajadhyaksha, "Faster path-based algorithm for traffic assignment," Transportation Research Record, no. 1443, pp. 75-83, 1994.

[27] T. Larsson and M. Patriksson, "Simplicial decomposition with disaggregated representation for the traffic assignment problem," Transportation Science, vol. 26, no. 1, pp. 4-17, 1992.

[28] C. Sun, R. Jayakrishnan, and W. K. Tsai, "Computational study of a path-based algorithm and its variants for static traffic assignment," Transportation Research Record, no. 1537, pp. 106115, 1996.

[29] M. Tatineni, H. Edwards, and D. Boyce, "Comparison of disaggregate simplicial decomposition and Frank-Wolfe algorithms for user-optimal route choice," Transportation Research Record, no. 1617, pp. 157-162, 1998.

[30] D. P. Bertsekas, "On the Goldstein-Levitin-Polyak gradient projection method," IEEE Transactions on Automatic Control, vol. 21, no. 2, pp. 174-184, 1976.

[31] H. Gunn, "An introduction to the valuation of travel-time savings and losse," in Handbook of Transport Modelling, Elsevier Science, 2000.

[32] D. Brownstone and K. Train, "Forecasting new product penetration with flexible substitution patterns," Journal of Econometrics, vol. 89, no. 1-2, pp. 109-129, 1998.

[33] K. E. Train, "Recreation demand models with taste differences over people," Land Economics, vol. 74, no. 2, pp. 230-239, 1998.

[34] S. Algers, P. Bergstrom, M. Dahlberg, and J. L. Dillen, "Mixed logit estimation of the value of travel time," Uppsala-Working Paper Series 1998:15, 1998.

[35] S. Hess, M. Bierlaire, and J. W. Polak, "Estimation of value of travel-time savings using mixed logit models," Transportation Research A, vol. 39, no. 2-3, pp. 221-236, 2005.

[36] L. J. LeBlanc, E. K. Morlok, and W. P. Pierskalla, "An efficient approach to solving the road network equilibrium traffic assignment problem," Transportation Research, vol. 9, no. 5, pp. 309318, 1975.

[37] D. Bertsekas and R. Gallager, Data Networks, Prentice Hall, Englewood Cliffs, NJ, USA, 2nd edition, 1992. 


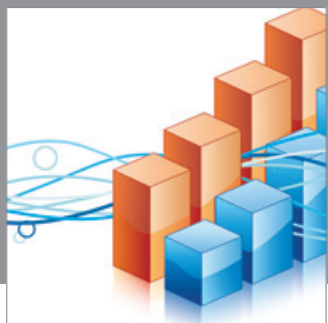

Advances in

Operations Research

mansans

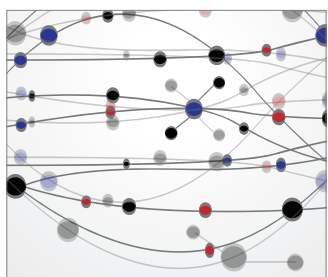

The Scientific World Journal
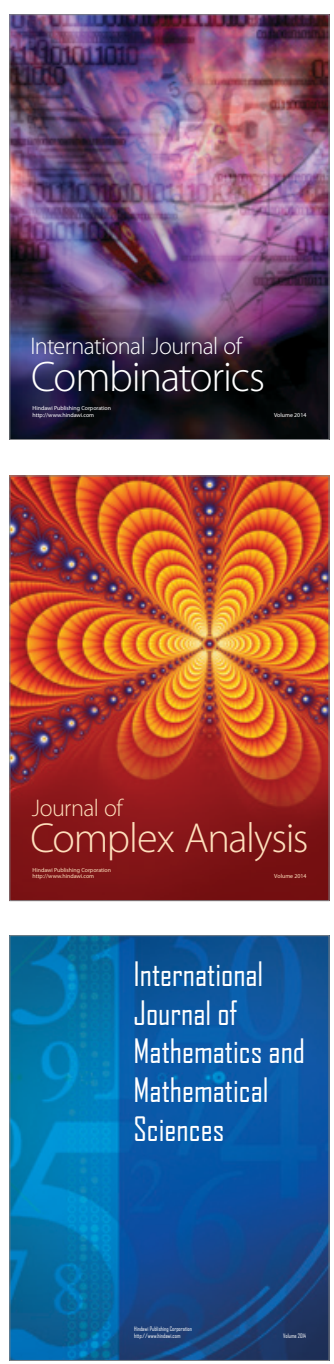
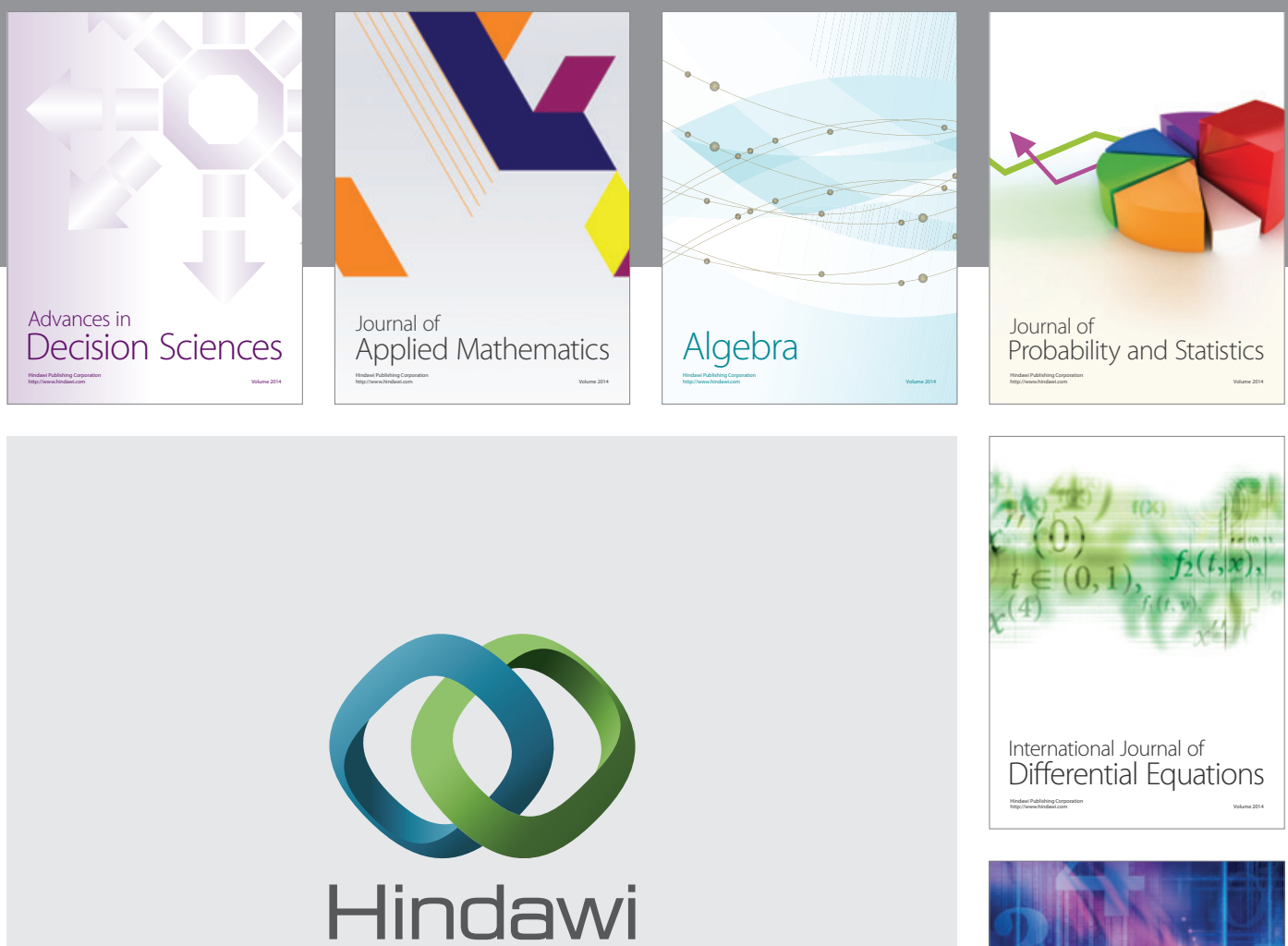

Submit your manuscripts at http://www.hindawi.com
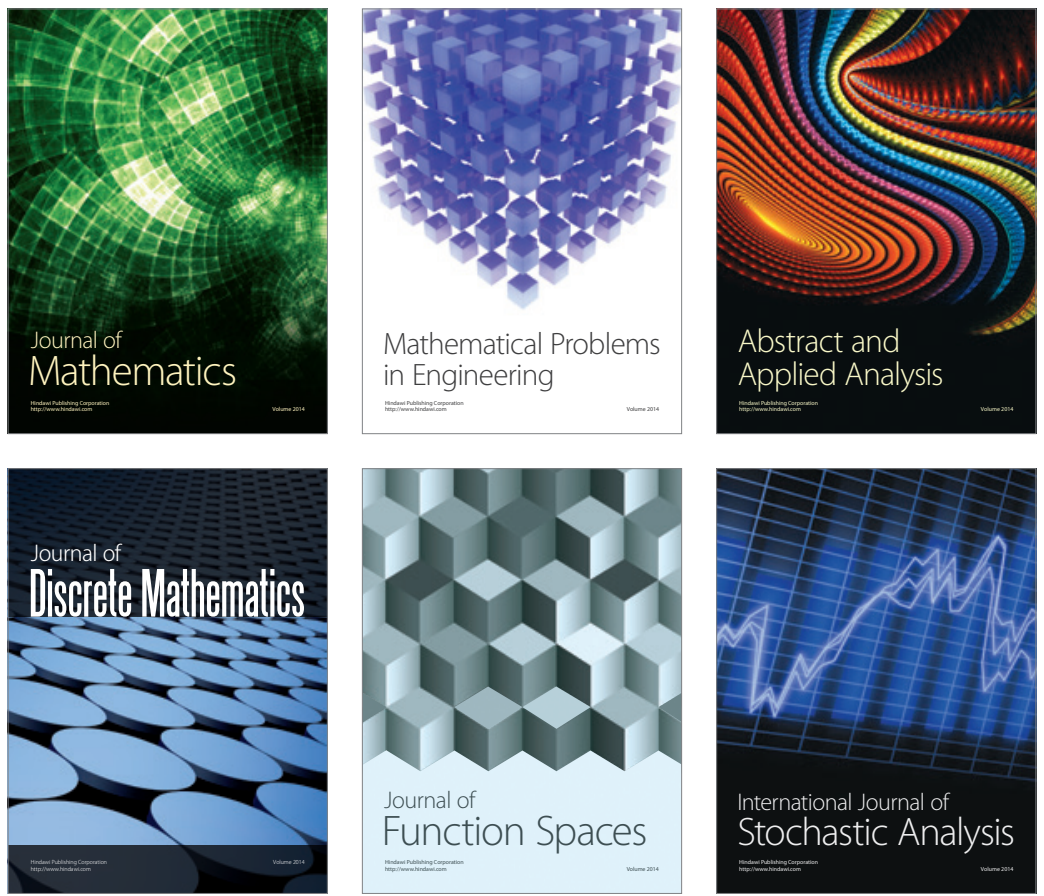

Journal of

Function Spaces

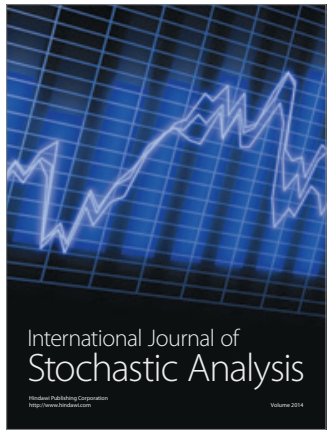

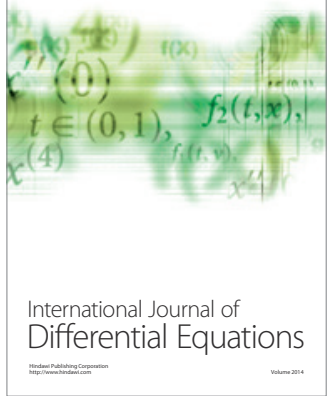
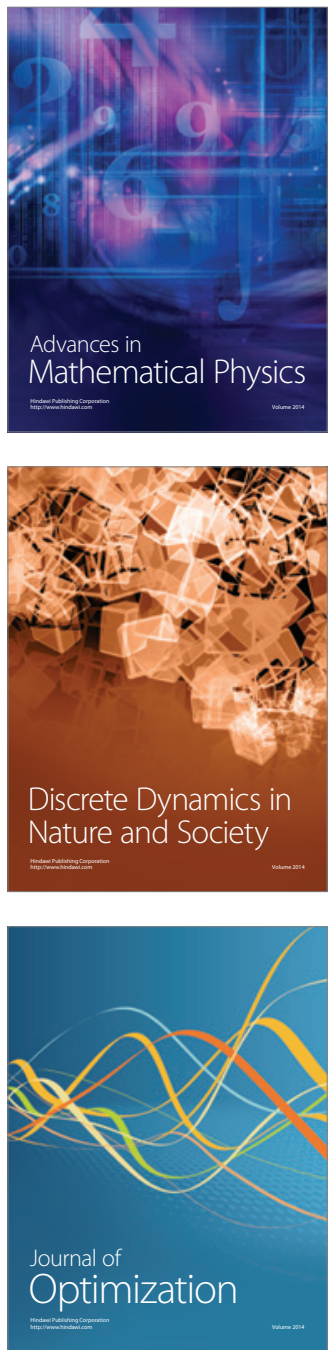